\title{
Specifics of joint deformation of fibered composite and homogeneouos materials
}

\author{
V. I. Galkin, A.R. Paltievich, E. V. Galkin * \\ Moscow Aviation Institute (National Research University), Volokolamskoe highway 4, Moscow, \\ 125993, Russia
}

\begin{abstract}
The article describes the possibility of usage of drawing method for production of pipes of fibered composite AL-B. The authors suggest using a construction of drawing mill, where the material volume reduction is carried out on the floating plug. With an aim of quality improvement technological shell is used. There were examined the mechanisms of joint deforming of compact blank part and solid shell.
\end{abstract}

\section{Introduction}

Force elements have a huge meaning in the construction of aircrafts. Their characteristic is exploitation in the conditions of cycle loading in high temperatures. Moreover, the construction weight is strictly regulated. Slitting reinforced pipes of fibered composite AL$\mathrm{B}$ as construction force elements are extensively used [1,2].

An item of composite material shall meet a series of requirements: it shall have a compact construction, a consistent connection between the components, shall keep continuity and solidness of fibers and have a high-grade surface.

A perspective method of production of fibered composite pipes production is drawing, which differs from other processes of fibered composite pipes production by local deformation zone. That is why the required force while drawing is considerably lower comparing to the processes of thermocompression bonding and gasostatic processing.

Task formulation

Drawing of fibered composite materials is conceptually different from technological processes of drawing of metals and alloys.

Fibered composite blank part has a fibered structure. The main task while composite blank part drawing is the production of compact structure items with a solid connection between components and keeping continuity of fibers. It is possible only in case when the compacting is realized in the conditions of hot deformation.

Fibered composite materials drawing is carried out with a joint deforming of die component and reinforcing fibers, all while the admissible level of stress is limited by solidness of fibers [2]. While the die part is deforming in the plastic strain region, the fibers should stay in the elastic field. While compacting composite fibered blank part, the decrease of its cross-axis size shouldn't lead to its elongation, which will lead to plastic

*Corresponding author: e.v.galkin@yandex.ru (tomd@mati.ru) 
deformation of fibers, which is unacceptable. The possibility of realization in practice of the process of drawing of pipes of fibered composite material is explained by the presence of reinforcing fibers in the blank part.

Either the pipes of fibered composite material are under exploitation or under production, the reinforcing fibers should receive the main strain in its axis direction. The presence of reinforcing fibers in the fibered composite material allows to elaborate the new technological process of production of direct-axis reinforced pipes by hot drawing.

Task formation and solution

The process of compacting is the main stage of production of items and blank parts of fibered composite material by solidphase methods. The most rational way to analyze the process of compacting is mathematical modelling.

It is suggested that drawing of fibered composite material should be conducted on floating plug, which will provide supporting of composite blank part material during the deformation process (Fig. 1). The plug represents a natural instrument for blank part composition, which guarantees the creation of the needed internal pipe diameter. [2].

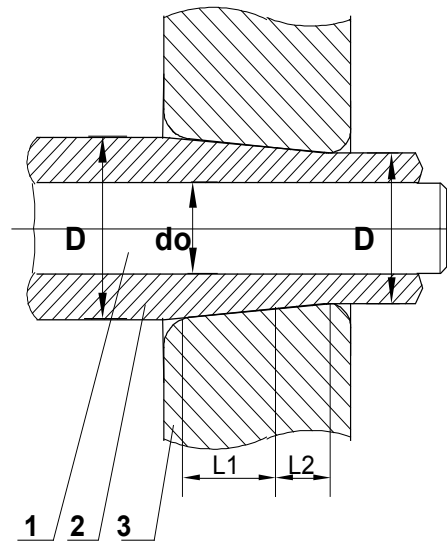

Fig. 1. Scheme of production of pipes of fibered composition material by drawing. 1 - plug; 2 - fibered composition material blank part; 3 - die block.

During hot drawing of fibered composition material blank parts there are two coneshaped zones of deformation. In the first zone which has length L1 (Fig. 1) material compacting is realized, in the 2 zone with length L2 is provided an additional soaking of compact material under strain.

The quality of surface of the produced item is connected with the conditions of contact friction between blank part and die. Unfavorable boundary conditions, specific for hot drawing, lead to an abnormally high wearing out of instrument, which provokes scratch marks on the item surface. In order to minimize shear stress on the external surface of the fibered composition material it is suggested to apply drawing with aluminum technological shell. This method allows to decrease the level of friction on the blank part external surface and increase the quality of produced item. Nevertheless, it is important to consider that the compacting of fibered composition material pipe blank part is accompanied with radial draft of material without its axis elongation, while the moldability of technological shell is carried out both in radial and axis directions, meaning that its length changes during deformation. The aim of modelling is the definition of process conditions in the 1 deformation zone, which would lead to minimal change of axis shell size. It is possible due to increase of cross-axis shell size during its moldability. 
Technological shell and instruments at the beginning and at the end of compacting process are represented on Fig.2.

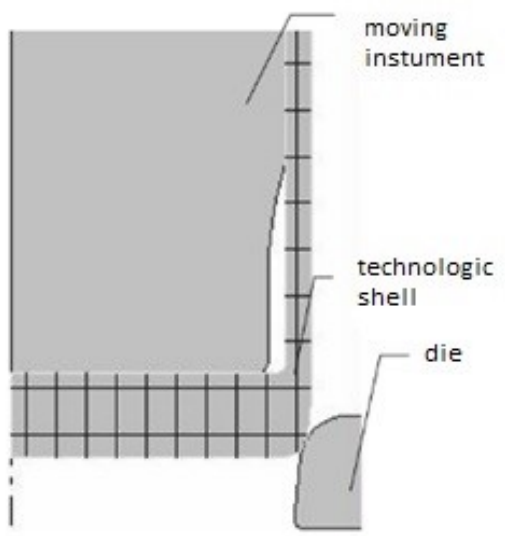

a)

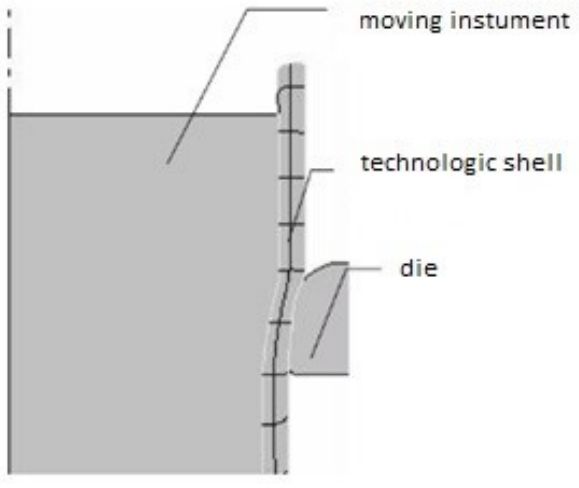

b)

Fig. 2. Deformation of technological shell during drawing process, a) - initial drawing conditions, b) - die cavity fulfilment during modelling.

Due to the fact that the deformation of porous material cannot be modelled by means of modelling program, the bottom part of internal die has cone form for the calculation of absolute cobbing of fibered composition material blank part. During the process of modelling this cone cavity is fulfilled with technological shell material, in the meantime cobbing strains are calculated (Figure 2b). The level of strains between die and technological shell corresponds to cobbing strains exposed on fibered composition material.

According to the research, presented in resource [2], the angle of conicity shouldn't exceed 5-10 $\square$. In such a case the values of extension strains and bending moment, which are exposed on fibers, are not big and they can be ignored in the zone of compacting.

Experimental solution proof

Modifying the angle of conicity and modelling the technological process, it is possible to monitor the value of strains on the internal boundary of technological shell. The material compacting is realized under its loading by external pressure of $12 \mathrm{MPag}$. The length of 1 deformation zone L1 is defined by the achievement of correspondent strain level (Fig. 3a).

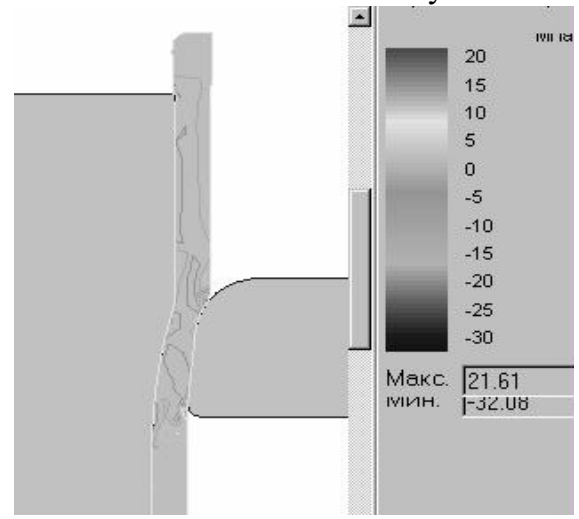

a)

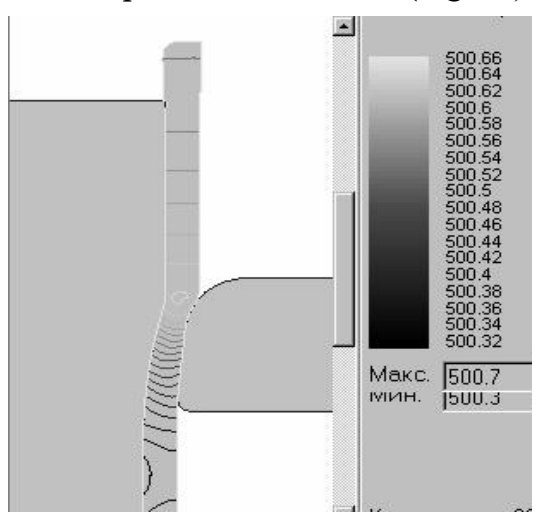

b)

Fig. 3. Allocation of strains and temperatures in the technological shell during the process of drawing. Bulbs of strains (a) and temperatures (b) in technological shell. 
The process of drawing is carried out in isothermal conditions at the temperature of $500^{\circ}$ $\mathrm{C}$, which is considered in the model [3]. The temperature at the end of technological process is shown on Fig. $3 b$.

The conducted modelling with different values of conicity angle of the draw die allows to receive numeric values, connecting the conicity angle with the elongation of the 1 deformation zone (Table 1).

Table 1. Consolidated values of draw die angle and corresponding elongation of the 1 deformation zone

\begin{tabular}{|c|c|c|c|c|c|c|c|c|c|c|}
\hline Draw die angle values, $^{\circ}$ & 1 & 2 & 3 & 4 & 5 & 6 & 7 & 8 & 9 & 10 \\
\hline L1, mm & 20,05 & 10,02 & 6,68 & 5,01 & 4,00 & 3,33 & 2,85 & 2,49 & 2,21 & 1,98 \\
\hline
\end{tabular}

Drawing according to elaborated regimes provided the production of pipes with compact structure with solid connection between components and maintenance of solidness of fibers.

The properties of materials, produced with the new technology, correspond to the requires ones. (Table 2).

Table 2. Mechanical properties of pipes of system Al-B with diameter of $20 \mathrm{~mm}$ and wall thickness of $1 \mathrm{~mm}$ with different contents of components volume concentration.

\begin{tabular}{|c|c|c|}
\hline Volume concentration of fibers & $\sigma_{\mathrm{b}}$, MPag & E, MPag \\
\hline 0,25 & $780-810$ & $140000-145000$ \\
\hline 0,35 & $1030-1070$ & $180000-190000$ \\
\hline 0,45 & $1280-1310$ & $200000-215000$ \\
\hline
\end{tabular}

As the result, the application of modern methods of technological processes elaboration and program means of mathematical modelling allows to receive new solutions at the stage of design and production engineering, which guarantee a considerable economy of material and timing production budget.

\section{References}

1. A.R. Paltievich, E.V. Preobrazhensky. Features of Technological Process Design via Mathematical Simulation Methods to Manufacture Al-B Fibrous Composite Products. // Scientific papers. (MATI Journal). 2010. № 17 (89). P. 47-51.

2. A.R. Paltievich, Sokolov A.V., Mathematical modelling of fibered composite material cell during assembled blank part compacting // Nonferrous metals 2003. № 5. P. 54-57.

3. V.I. Galkin, M.G. Golovkina, A.R. Paltievich, Forecasting of structural state and mechanical properties of aluminum alloys items with application of computer modelling // Aviation industry. 2015. № 1. P. 48-54. 